\title{
Adaptation and evaluation of a weak recycling strategy for inflow boundary layers in large-eddy simulation
}

\author{
J. Boudet and A. Giauque \\ Univ Lyon, Ecole Centrale de Lyon, CNRS \\ LMFA, F-69130, ECULLY, France
}

April 22, 2020

\begin{abstract}
This article presents a detailed analysis of an improved weak recycling method used to generate turbulent inflow conditions for large-eddy simulations. Answering some of the points raised in the literature, various flat-plate cases are considered in order to evaluate the fidelity and the robustness of the method. It is shown that the distance it takes for the canonical compressible boundary layer to forget its weak recycling inflow turbulence is rather short $\left(<10 \delta\right.$, for $\operatorname{Re}_{\theta}$ up to 2340$)$. Yet, the study also reveals that the optimal distance between the inlet and the source plane location used to extract the fluctuations depends on the case at hand with a tendency of compressible simulations to require a sightly larger distance. Cases involving compressibility effects $(M=0.8)$ and 3D effects (wall in translation perpendicular to the main flow) show that the recycling method remains effective in more complex flows. When using limited resolution inlet flow profiles coming from experiments as an input, spurious fluctuations are observed whose main wavelength equals the recycling distance. This study proposes a strategy to circumvent this drawback by limiting the amplitude of recycled velocity fluctuations with respect to the mean velocity at the inlet.
\end{abstract}

Keywords: Turbulent inflow; Recycling; Boundary layer; Large-eddy simulation. 


\section{Introduction}

For the past twenty years, in aerodynamics, there has been a strong research effort in order to bridge the gap between experiments and high-fidelity numerical techniques such as Large Eddy Simulation (LES). In a realistic framework, one of the key ingredients for such convergence is the proper imposition of the turbulent inflow boundary conditions. More specifically, simulated flows often involve incoming turbulent boundary layers (TBL), for example at hub or casing in turbomachines.

Recently, progress and remaining challenges in turbulent inflow conditions have been reviewed in detail by $\mathrm{Wu}$ [1]. To sum up, numerous approaches have been proposed that can be organized into three main families:

- Full approach.

The entire development length of the incoming TBL is simulated. As shown by Gao et al. [2], the simulation can be limited to a narrow width (some $\delta$ 's) with periodicity, given the small transverse length-scale. However, the streamwise length of the TBL simulation necessary to reach a given value of $\theta$ varies as $R e_{\theta}^{1 / 4} \cdot \theta$.

- Synthetic inflow turbulence approach.

One of the most advanced representative of this family of methods has been proposed by Jarrin and co-authors in 2006 [3] and is called SEM for Synthetic Eddy Method. It has been further developed by Pamies et al. [4] and used in the context of Combustion [5] or Computational Aero-Acoustic (CAA) simulations [6]. Developments have been proposed recently by Xie et al. [7]. The main drawback of this approach however is that it can be difficult to impose prescribed mean flow velocity profiles while at the same time introducing artificial structures that develop rapidly downstream into a realistic turbulent boundary layer.

- Weak recycling approach.

Weak recycling methods can be traced back to the work of Wu and co-authors in 1995 [8]. They have been formalized by Lund et al.[9] in 1998. Since then, these methods have been extended to compressible flows by Stolz \& Adams [10]. Sagaut et al. [11] provide an interesting review of the weak recycling approaches for compressible flows. In this approach, the turbulent structures are bred by the 3D unsteady solver and can develop realistic features rapidly. 
The present article focuses on this last family of methods, introduces new developments, and intends to answer some of the points raised by $\mathrm{Wu}[1]$ in his review. A compressible extension of the model (as presented by Sagaut et al. [11]) is investigated for various flat-plate cases in order to evaluate the adaptation and robustness of the method for turbomachinery configurations. More specifically, the distance it takes for the canonical compressible boundary layer to forget its weak recycling inflow turbulence is investigated. The capability of the approach to accurately capture 3D effects related to a turbulent boundary layer developing over a moving wall is also addressed. Finally, a more realistic case is analyzed in which the incoming boundary layer is known from an experimental velocity profile whose resolution is lower than the simulation. In this context, best practices are discussed in order to deal with the non-zero streamwise correlation between the source and target plane sections.

By comparison with the existing literature, the novelty of the present study essentially lies in the following features:

- User-defined mean-velocity profiles are used at the inflow, and the sensitivity of the method to the profile description is evaluated.

- An exponential averaging strategy is proposed to extract the fluctuations on the source plane, while the simulation is running.

- A limitation of the fluctuations is proposed and tested, with the objective to limit the development of periodic perturbations.

- The sensitivity of the method to the length of the recycling zone is investigated.

\section{Formulation}

The weak recycling methodology relies on two flow sections:

- The inflow boundary condition, or target section, where turbulent conditions have to be imposed, conforming to prescribed mean profiles.

- The source section, downstream, where fluctuations are extracted.

Inflow $=$ target section $\quad$ In the present approach, the mean velocity profiles $\left(\bar{u}_{i, t g t}\left(y^{+}\right)\right.$, $i \in\{1,2,3\})$ are specified by the user at the inflow. For example, they can be designed from available experimental data. 
During the computation, rescaled fluctuations from the source section $\left(u_{i, s r c}^{\prime}, i \in\{1,2,3\}\right)$ are superimposed. Precisely, the instantaneous velocity to be imposed at the inflow is expressed as:

$$
\begin{aligned}
\forall i \in\{1,2,3\}: \quad u_{i, t g t}(y, z, t) & =\bar{u}_{i, t g t}(y) \\
& +(1-W(\eta)) \cdot \beta \cdot u_{i, s r c}^{\prime}\left(y^{+}, z, t\right)+W(\eta) \cdot \beta \cdot u_{i, s r c}^{\prime}(\eta, z, t)
\end{aligned}
$$

where $y^{+}=y \cdot u_{w, t g t} / \nu$ and $\eta=y / \delta_{t g t}$ are the inner and outer coordinates. The rescaling factor is calculated as: $\beta=\left(\delta_{s r c} / \delta_{t g t}\right)^{1 / 8}$, according to Stolz and Adams [10]. Limiting the implementation to cases where the mean axial velocity largely dominates the other velocity components, $\delta$ and $u_{w}$ are here computed using only the axial mean velocity profile. The influence of this choice could be analyzed in future studies. Finally, the weighting function between the inner and outer regions is:

$$
W(\eta)=\frac{1}{2}\left[1+\tanh \left(\frac{\alpha(\eta-b)}{(1-2 b) \eta+b}\right) / \tanh (\alpha)\right]
$$

with $\alpha=4$ and $b=0.2$.

Density and temperature are rescaled accordingly:

$$
\begin{aligned}
& \rho_{t g t}(y, z, t)=\bar{\rho}_{t g t}(y)+(1-W(\eta)) \cdot \beta \cdot \rho_{s r c}^{\prime}\left(y^{+}, z, t\right)+W(\eta) \cdot \beta \cdot \rho_{s r c}^{\prime}(\eta, z, t) \\
& T_{t g t}(y, z, t)=\bar{T}_{t g t}(y)+(1-W(\eta)) \cdot \beta \cdot T_{s r c}^{\prime}\left(y^{+}, z, t\right)+W(\eta) \cdot \beta \cdot T_{s r c}^{\prime}(\eta, z, t)
\end{aligned}
$$

In order to limit the amplification of periodic perturbations by the recycling, a ceiling strategy has been implemented and evaluated. The ceiling on the velocity perturbations is defined as:

$$
\forall i \in\{1,2,3\}:\left|u_{i, t g t}(y, z, t)-\bar{u}_{i, t g t}(y)\right| \leq \chi_{c e i l} \times \sqrt{\sum_{i=1}^{3} \bar{u}_{i, t g t}^{2}(y)}
$$

$\chi_{c e i l}$ can be interpreted as an instantaneous turbulent intensity ceiling. In the present study, $\chi_{\text {ceil }}=0.2$. 
Source section This section is located downstream of the inflow, at several $\delta_{t g t}$ (the influence of the distance from the inflow is investigated thereafter). In order to extract the fluctuations $\left(u_{i, s r c}^{\prime}=u_{i, s r c}-\bar{u}_{i, s r c}, i \in\{1,2,3\}\right)$, the mean velocity components $\left(\bar{u}_{i, s r c}\right.$, $i \in\{1,2,3\})$ have to be extracted and updated. A combination of a lateral spatial average $($ noted $\langle$.$\rangle ) and a temporal exponential average [12] is used:$

$$
\forall i \in\{1,2,3\}, y, n \geq 0: \quad \bar{u}_{i, s r c}^{n+1}(y)=\left(1-c_{\text {exp }}\right) \bar{u}_{i, s r c}^{n}(y)+c_{\text {exp }}<u_{i, s r c}^{n+1}>(y)
$$

where the coefficient $c_{\text {exp }}$ controls the cut-off frequency $f_{c}$ of the exponential low-pass filtering:

$$
c_{\exp } \approx 3.628 f_{c} \Delta t
$$

and $f_{c}$ is calculated from the inflow conditions: $f_{c}=u_{w, t g t} / \delta_{t g t}$, which yields:

$$
c_{\exp }=\frac{3.628 u_{w, t g t} \Delta t}{\delta_{t g t}}
$$

Excitation In order to accelerate the development of the turbulent fluctuations by the recycling procedure, a source term roughness is positioned between the target and source planes. This excitation is transitory. In the present work, it is applied during the first 100000 iterations only.

The formulation of the source term roughness was introduced in [13], where it was employed to trigger the transition in a laminar boundary layer. The drag force per unit volume expresses:

$$
\frac{-1}{2 l_{x}} \rho C_{D}\|\mathbf{u}\| \mathbf{u}
$$

where $l_{x}$ is the streamwise length of the roughness and $C_{D}=1$.

In the present work, the source term is applied to 5 cells in the streamwise direction (thus $l_{x}=5 \Delta x$ ), over the full width of the computational domain. We propose to set the height of the roughness to $\delta^{*}$. This order of magnitude is consistent with the measurements of Dryden [14] (cited by Cousteix [15]), for a transition at the position of the roughness. Since this procedure is transitory, a more precise tuning of the roughness height does not appear necessary. 


\section{$3 \quad$ Evaluation with numerical inflow profiles (NIP)}

As already pointed out, the present study is originally devoted to turbomachinery applications. In such configurations, inflow conditions are generally to be imposed upstream of blade rows, in regions of mild pressure gradient and moderate wall curvature (hub or casing curvature radius is usually one or two orders of magnitude larger than the boundary layer thickness). However, the boundary layer can be developing over a moving wall and transonic flow conditions are common. Consequently, the method is evaluated on a flat plate geometry, in various conditions:

- Incompressible $(M=0.2)$ axial case.

- Transonic $(M=0.8)$ axial case.

- Transonic $(M=0.8)$ case with moving wall.

Even though the present evaluation was conceived to be representative of turbomachinery conditions, its diversity makes it appropriate for other engineering applications. For example, transonic flow conditions and 3D boundary layers are usual in aircraft aerodynamics.

In the present section, simulations with recycling inflow ("short simulations") are compared to simulations of the full-length boundary layer (i.e. starting from the leading edge). This is sketched in Fig.1. The mean velocity profile of the recycling simulation is provided by the full-length simulation, and is thus perfectly adapted since it is a solution of the same solver on the same grid. Such a profile is referred to as "numerical inflow profile" (NIP). The use of "imperfect" inflow profiles will be investigated in the next section.

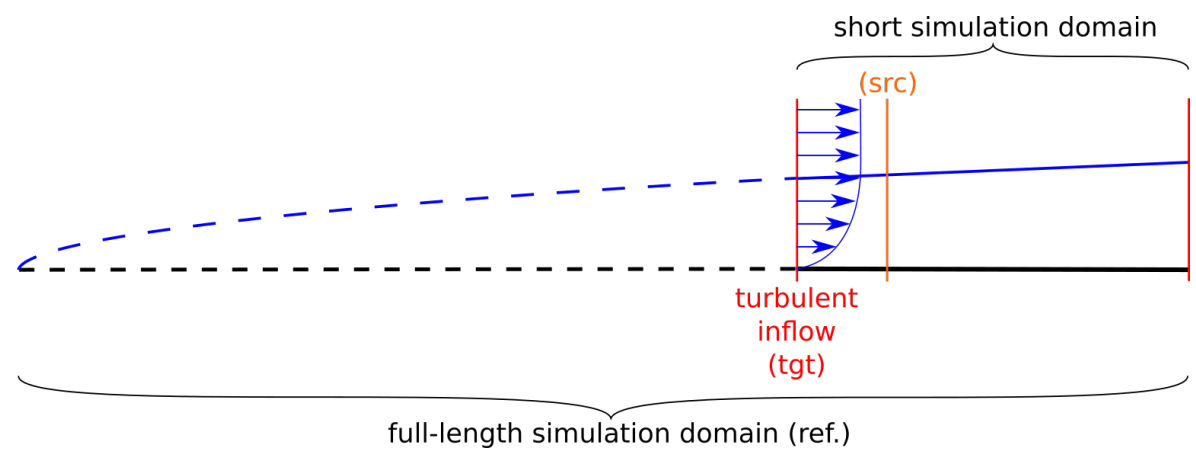

Figure 1: Evaluation with a numerical inflow profile (NIP).

The simulations are carried out with the in-house solver Turb'Flow [13, 2]. The finite volume discretization uses a four-point centered interpolation for the inviscid fluxes, with 
fourth-order artificial viscosity (coefficient: 0.004, definition in [13]). A two-point centered interpolation is used for the viscous fluxes. Time marching uses a 3-step Runge-Kutta.

Fluid is modeled as ideal gas $(P=\rho r T$, with $r=287 \mathrm{~J} / \mathrm{kg}-\mathrm{K})$, with a constant dynamic viscosity $\mu=1.81 \cdot 10^{-5} \mathrm{~kg} / \mathrm{m}$-s. The subgrid-scale viscosity is evaluated with the WALE model [16]. In order to reach the objective value of $R e_{x}=1.3 \cdot 10^{6}$, the boundary layer has to develop over $L_{x}=0.3 \mathrm{~m}$. The height of the computational domain (normal to the wall) is $L_{y}=14.8 \cdot 10^{-3} \mathrm{~m}$, corresponding to about $2.6 \delta$ at the outlet. The width of the computational domain is: $L_{z}=5.9 \cdot 10^{-3} \mathrm{~m}$, corresponding to about $1.2 \delta$ at the outlet. In the full-length simulations, a source-term trip is set at $x=0.066 \mathrm{~m}$ to force the transition to turbulence, using the methodology presented in [13]. In the turbulent region, cell sizes at the wall, in wall units, satisfy: $\Delta x^{+}<80$ (streamwise), $\Delta y^{+}<2$ (wall-normal) and $\Delta z^{+}<30$ (cross-stream). Grid is uniform along $x$ and $z$, and expansion ratio is 1.15 along $y$. The total number of grid points is $1.43 \cdot 10^{6}$ for the full-length simulation, and $0.5 \cdot 10^{6}$ for the short simulation with recycling. Time step is $4 \cdot 10^{-8} \mathrm{~s}$. Density and velocity components are imposed at the inflow. Adiabatic no-slip is prescribed at the wall. Pressure is imposed on the upper boundary and at the outflow, with a partially non-reflecting condition. Periodicity is imposed on the lateral boundaries.

\subsection{Incompressible $(M=0.2)$ case}

The first test-case is an incompressible boundary layer. At the inflow: $U_{e}=70 \mathrm{~m} / \mathrm{s}$, $\rho=1.177 \mathrm{~kg} / \mathrm{m}^{3}$ and $T=300 \mathrm{~K}$, thus $M=0.2$.

Two simulations have been carried out: (i) a reference full-length simulation, and (ii) a simulation with a rescaled inflow at $x_{1}=0.2 \mathrm{~m}\left(\operatorname{Re}_{\theta}=1430\right)$. For the second simulation, the mean velocity profile imposed at the inflow is extracted by averaging the full-length simulation at the same position. The source section of the recycling is positioned at $x_{s r c}=x_{1}+2 \delta\left(x_{1}\right)$.

Fig.2 shows the streamwise evolution of the friction coefficient $C_{f}$, for the two simulations. The reference LES (full length) is shown to follow the laminar evolution up to the trip at $x=0.066 \mathrm{~m} \approx 20 \delta\left(x_{1}\right)$, and the turbulent evolution downstream. The short simulation, with the recycling inflow condition, performs very well. Only a slight decrease of friction is observed after the inlet at $x_{1} / \delta\left(x_{1}\right)=62$. Downstream, the levels are in very 
good agreement with the reference simulation.

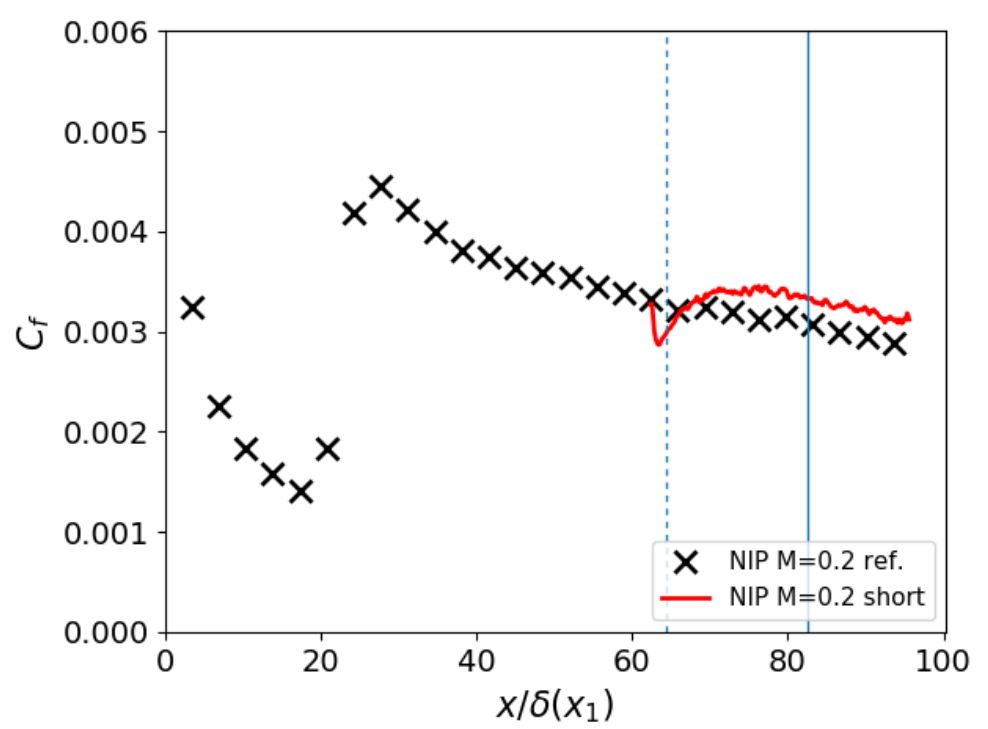

Figure 2: NIP $M=0.2$ case: streamwise evolution of $C_{f}$. Symbols: reference full-length simulation, line: short simulation with recycling inflow. The vertical thin lines locate $x_{s r c}$ (dashed line) and the profile extraction plane (continuous line).

The vertical profiles of mean and fluctuating velocities, at $x=x_{1}+20 \delta\left(x_{1}\right)$, are shown in Fig.3. The corresponding abscissa is indicated by a thin continuous line in Fig.2. A very good prediction of the mean velocity profile is achieved by the short simulation. Regarding the fluctuations, a very good agreement is also observed on $v^{\prime}$ (circles and dashed line) and $w^{\prime}$ (triangles and dotted line). Concerning the streamwise component $u^{\prime}$ (crosses and continuous line), a slight overestimate is observed in the short simulation.
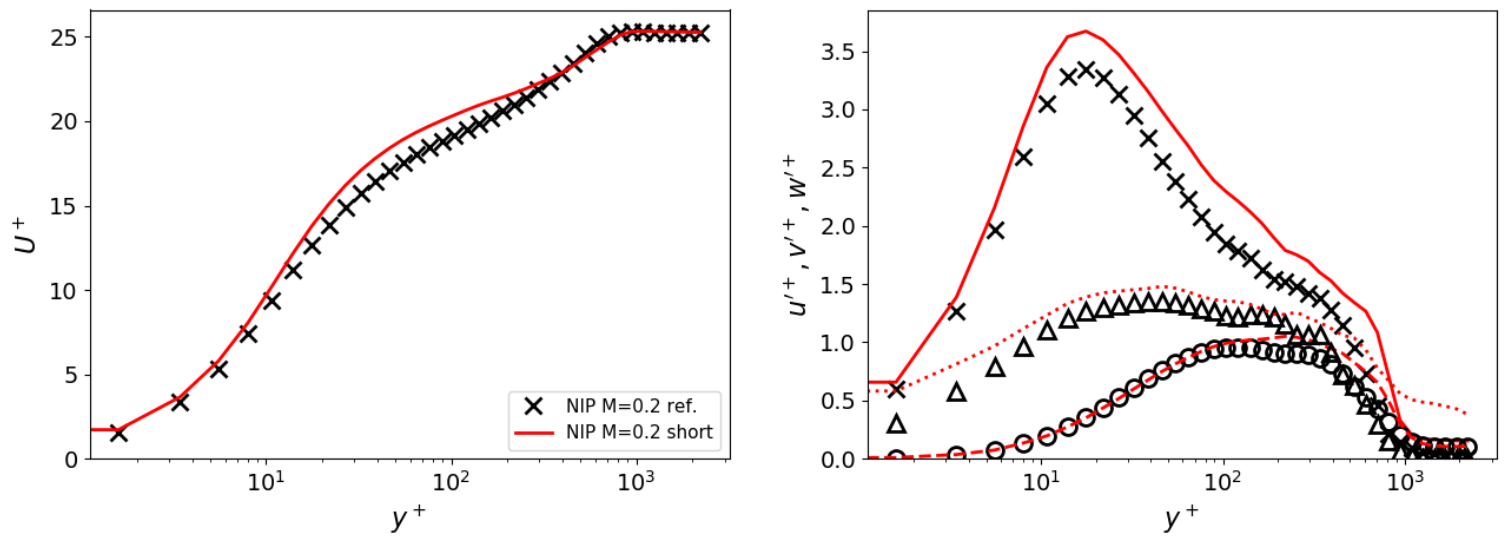

Figure 3: NIP $M=0.2$ case: profiles of mean and fluctuating velocities at $x=x_{1}+20 \delta\left(x_{1}\right)$. Symbols: reference full-length simulation, lines: short simulation with recycling inflow. Right plot: $u^{\prime}(\times,-), v^{\prime}(\circ,---)$ and $w^{\prime}(\Delta, \cdots)$. 


\subsection{Transonic $(M=0.8)$ boundary layer over a stationary wall}

In this case the flow develops again a boundary layer over a stationary wall, but differs from the previous incompressible one because of its inflow Mach number. The inflow velocity and density, and the viscosity, are kept identical to the previous subsonic case, ensuring that the Reynolds Number remains the same. In this context, and in order to reach an inlet flow Mach number of 0.8, the temperature is decreased to $19.05 \mathrm{~K}$ and the pressure to $6435 \mathrm{~Pa}$, artificially decreasing the sound speed to $87.5 \mathrm{~m} / \mathrm{s}$.

As for the previous case, two sets of simulations are systematically carried out: (i) a reference simulation of the full length, and (ii) simulations with a rescaled inflow at $x_{1}=0.2 \mathrm{~m}$.

\subsubsection{Influence of the source plane section location}

As pointed out by $\mathrm{Wu}[1]$ in his review, the question of the influence of the location of the source plane for the recycling methodology remains an open issue. In order to shed some light, four simulations are carried out in this case. All four use the same parameters, except they differ from each other by the axial distance between the source and target planes. Four distances are considered: $2 \delta, 6 \delta, 10 \delta$ and $14 \delta$. Results are compared with the ones obtained in the full length case.

Figure 4 compares the evolutions of the friction coefficient. The full-length simulation is plotted as reference. The oscillation observed for $10<x / \delta\left(x_{1}\right)<20$ corresponds to the tripping band, used to enforce transition to turbulence. Concerning the simulations using the turbulent inlet forcing, one observes that except for the case in which the source plane is the closest, at $2 \delta$, the friction coefficient is very well recovered.

Figure 5 compares the velocity profiles for $U^{+}, u^{\prime+}, v^{\prime+}$ and $w^{\prime+}$, at $x=x_{1}+20 \delta\left(x_{1}\right)$, for the different simulations. It appears the four simulations with recycling properly reproduce the reference velocity profiles (mean and fluctuations). Only small discrepancies appear for the case having the smallest recycling distance $2 \delta$. They are visible in the log region and in the external region of the turbulent boundary layer. Yet, larger discrepancies are observed in the fluctuation profiles above the boundary layer for all cases. They are related to a non zero fluctuating velocity (though quite small) in the external flow that could be linked to acoustic perturbations introduced by the recycling methodology. 


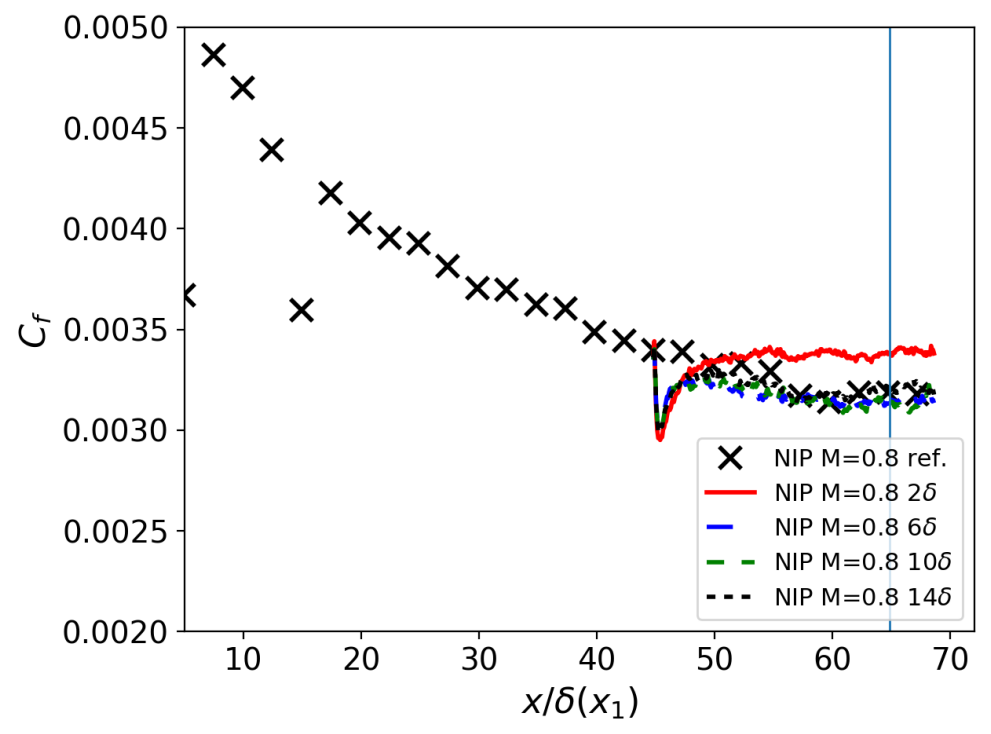

Figure 4: NIP $M=0.8$ case, influence of the source plane location: friction coefficient. Symbols: reference full-length simulation, lines: short simulations with recycling inflow. The vertical continuous thin line locates the profile extraction plane at $x=x_{1}+20 \delta\left(x_{1}\right)$.
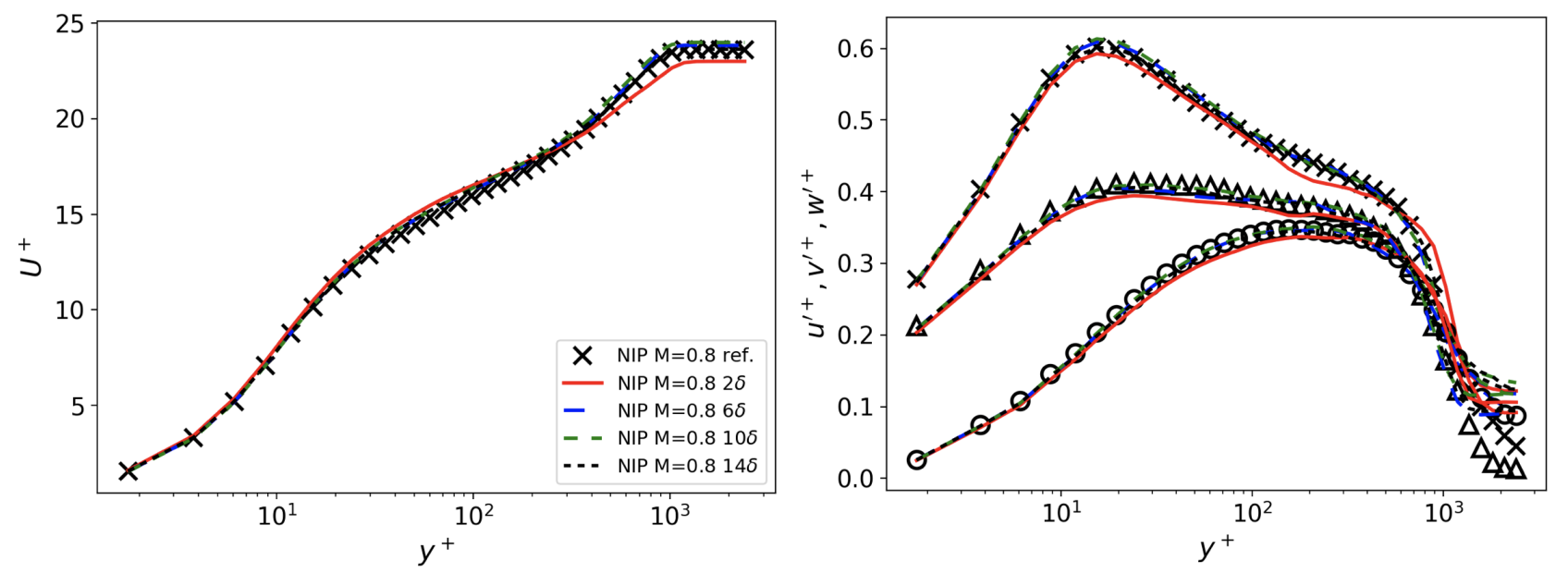

Figure 5: NIP $M=0.8$ case, influence of the source plane location: wall-normal evolution of $U^{+}$and $u^{\prime+}, v^{\prime+}, w^{\prime+}$ at $x=x_{1}+20 \delta\left(x_{1}\right)$. Symbols: reference full-length simulation, lines: short simulations with recycling inflow. Right plot symbols: same conventions as Fig. 3. 


\subsection{Transonic $(M=0.8)$ boundary layer over a moving wall}

In this case the flow develops a turbulent boundary layer over a wall in translation at $M=0.5$ in a direction perpendicular to the inflow velocity (along the $z$ direction). This case is expected to emphasize the influence of $3 \mathrm{D}$ effects on the developpement of the boundary layer downstream of the inlet. The underlying question is whether the turbulence recycling method is appropriate in a 3D boundary layer, for example in a turbomachinery case where the hub is rotating.

Figure 6 shows the evolution of the friction coefficient with respect to the distance. A significant difference is found whether the distance between the source and target planes is set to $2 \delta$ or $10 \delta$. The friction coefficient is significantly underpredicted in the $2 \delta$ case and only recovers at the outlet the value obtained in the full length case. For the $10 \delta$ case, friction is slightly overpredicted.

Figure 7(left) compares the boundary-layer profiles for $U^{+}$and $W^{+}$. It shows that the recycling methodology performs slightly better in the $10 \delta$ case. When the source plane is at $2 \delta$ of the target plane, $U^{+}$is underestimated in the inner part of the boundary layer and overestimated above. As for $W^{+}$, it is overestimated in the $2 \delta$ case. Figure 7 (right) provides the additionnal comparison of the boundary-layer profiles for $u^{\prime+}, v^{\prime+}$, and $w^{\prime+}$. All three plots show the same tendency for the recycling methodology to correctly reproduce the evolution of the fluctuating velocities in the boundary layer regardless of the distance between the source and target planes. However, one observes much larger fluctuation levels outside of the boundary layer when the distance between the two planes is reduced. This is probably related to additional acoustic fluctuations generated by the forcing in this case.

\section{Evaluation with an experimental inflow profile (EIP)}

The previous simulations can be seen as ideal situations for the mean velocity profile at the inflow, since the mean profile always matches the solution of the solver on the same grid. This allowed direct comparisons with the full-length simulations. However, practical situations are not as favorable, for example when the velocity profile comes from experimental measurements with a limited resolution. The present section aims at 


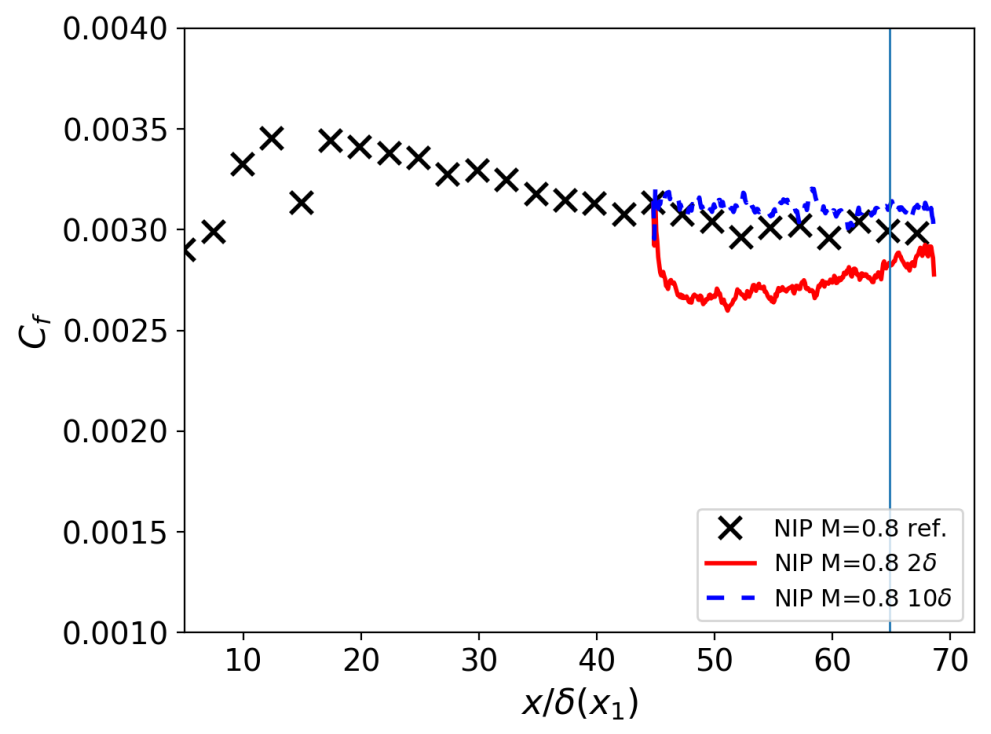

Figure 6: NIP $M=0.8$ 3D case: friction coefficient. Symbols: reference full-length simulation, lines: short simulations with recycling inflow. The vertical continuous thin line locates the profile extraction plane at $x=x_{1}+20 \delta\left(x_{1}\right)$.
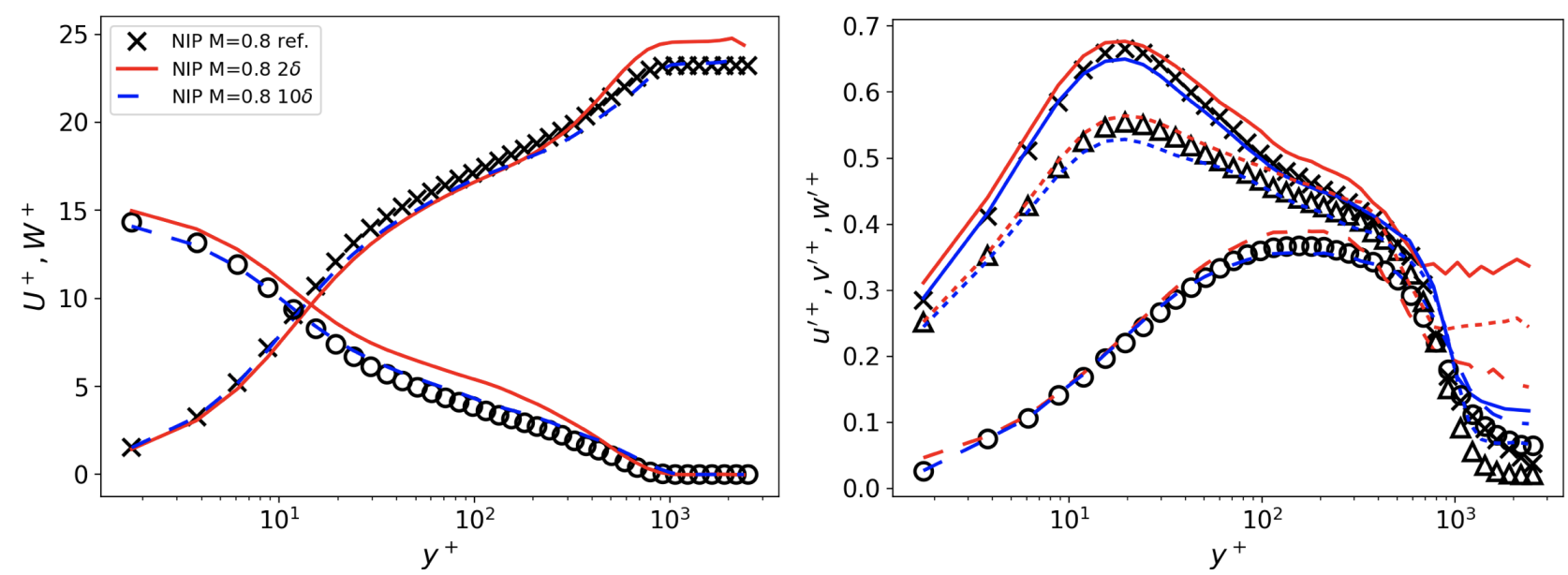

Figure 7: NIP $M=0.8$ 3D case: wall-normal evolution of $U^{+}$and $W^{+}$(left), $u^{\prime+}, v^{\prime+}, w^{\prime+}$ (right) at $x=x_{1}+20 \delta\left(x_{1}\right)$. Symbols: reference full-length simulation, lines: short simulations with recycling inflow. Right plot symbols: same conventions as Fig. 3. 
investigating the robustness of the methodology in such situations.

The present investigation relies on the boundary layer velocity profiles acquired by Jacob et al. [17] during the experimental study of a blade tip-leakage flow. Only the incoming boundary layer is considered in the present study. Velocity profiles are available at three abscissa.

It can be noted the whole blade tip-leakage flow (incoming boundary layer + blade) has already been simulated with zonal LES by Boudet et al. [18], computing the whole development of the incoming boundary layer over a length adapted to match the first velocity profile of the experiment. The present recycling methodology should allow a simulation starting at the first velocity profile, prescribed from the experiment, without the computation of the upstream development and without specific calibration.

The computational setup is sketched in Fig.8. The three sections correspond to the measurement positions. The flow upstream of section 1, through the wind tunnel apparatus, is not characterized experimentally. The width and height of the computational domain are respectively $0.01 \mathrm{~m}=1.3 \delta\left(x_{3}\right)$ and $0.02 \mathrm{~m}=2.6 \delta\left(x_{3}\right)$. The experimental mean velocity profile is imposed at section 1 (where $R e_{\theta}=2340$ ), with the recylcing inflow condition, and comparisons are performed between the computation and the experiment at the other two sections.

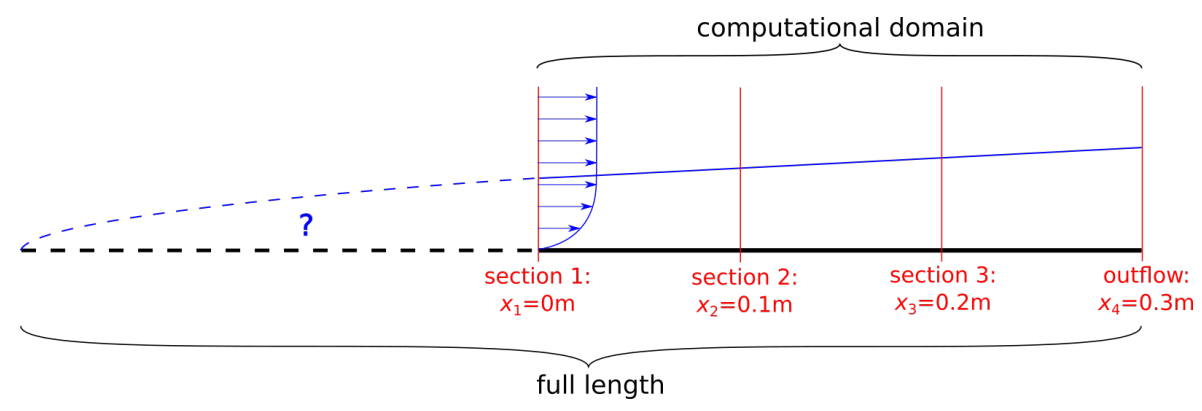

Figure 8: EIP case: computation setup.

Corrected mean velocity profile at inflow The experimental mean-velocity profile at section 1 is shown in Fig.9, with black crosses. Wall proximity affects the hot-wire measurements and results in surprisingly high values of velocity at low $y$. Consequently, these points need to be discarded and the following procedure is set-up to reconstruct the velocity profile. The friction velocity is determined by fitting Cole's wake law in the external region of the boundary layer. Then, Van Driest profile is constructed in the inner region of the boundary layer, and connected with the experimental results above $y^{+}=92$. 
The corrected velocity profile is shown with a black continuous line in Fig.9. A more rudimentary correction is also tested, with a simple linear evolution below $y^{+}=92$. The corresponding profile is also shown in Fig.9, with a black dashed line. This corresponds to the lowest resolution of the mean velocity profile.

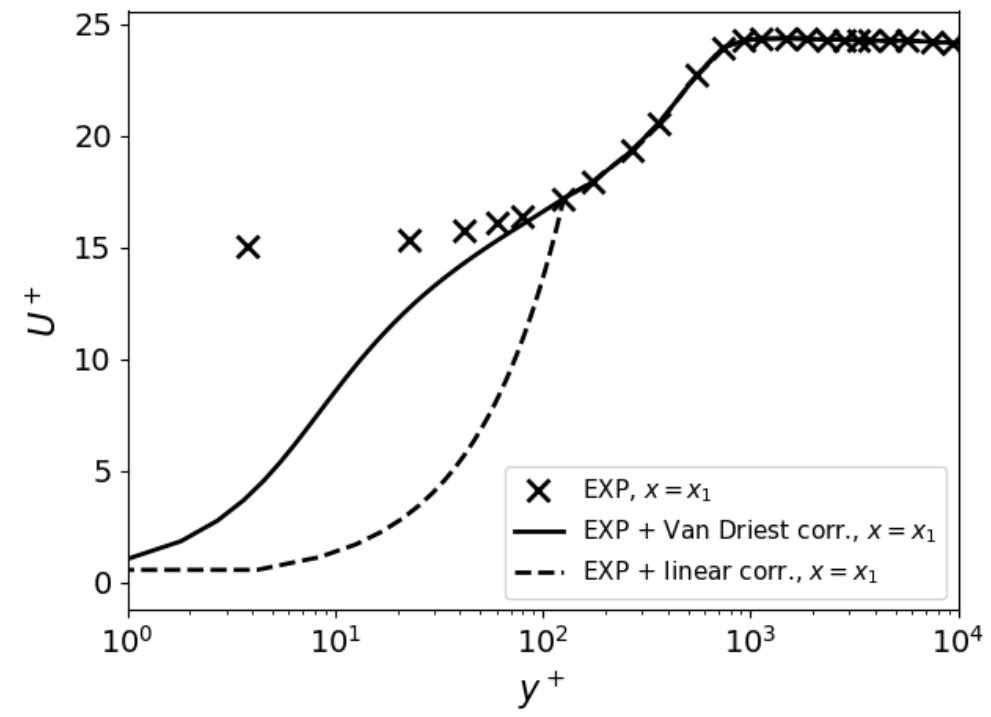

Figure 9: EIP case: mean velocity at inflow $\left(x=x_{1}\right) . \times$ : experiment, $\longrightarrow$ : experiment with Van Driest correction at wall, - - - : experiment with linear correction at wall.

\section{Influence of the methodology on the development of artificial periodic}

fluctuations In the present EIP case, periodic fluctuations are shown to develop because of the recycling. Given the approximately incompressible flow conditions, these fluctuations are particularly visible on density, as illustrated in Fig.10. These fluctuations show a dominant wavelength corresponding to the distance between the recycling planes. This suggests a coupling between the source and target planes. Such a coupling has already been observed, for example by Simens et al. [19] in their incompressible boundary layer simulations. Simens et al. relate this coupling to the lagrangian persistence of the large turbulent eddies, advected from the inflow to the source plane and rescaled back to the inflow. In the present compressible simulations, acoustic waves also contribute to this coupling, which is confirmed by the analysis of the unsteady flow fields. Simens et al. suggest to increase the recycling distance (some hundreds of $\theta$ ), when the physics of the boundary layer is to be investigated in details. In the present case, the objective of the methodology is to generate a "fairly physical" boundary layer to feed simulations of complex turbomachinery flows. Focus is not on the boundary layer physics, and a short 
recycling distance is sought. The methodology is to be evaluated with this perspective. Finally, Simens et al. observed an amplification of this coupling when the upper boundary of the computational domain is low, as in the present cases. This effect should be alleviated in pratical applications where the computational domain height is large compared to the boundary layer thickness.
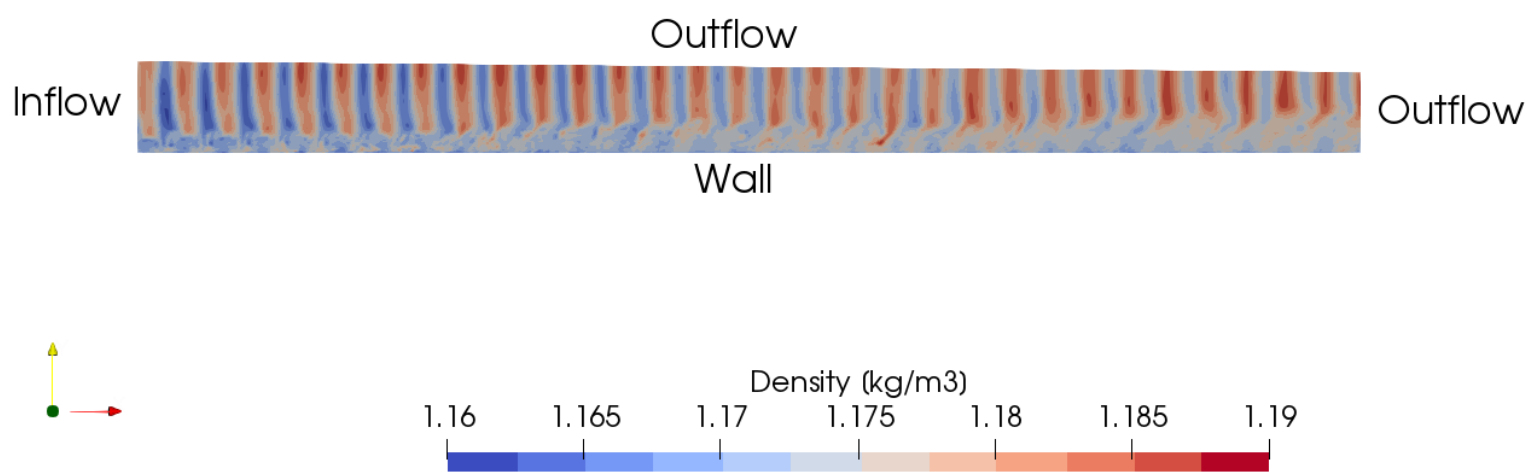

Figure 10: EIP ref. simulation: instantaneous density contours.

Different simulations have been performed to evaluate the sensitivity of these fluctuations to the parameters of the recycling. The simulation parameters are summarized in table 1. Furthermore, the last column of the table provides a measurement of the fluctuation intensity as: $\left(\rho_{\max }-\rho_{\min }\right) /\left(2 \rho_{0}\right)$, where $\rho_{\min }$ (resp. $\left.\rho_{\max }\right)$ is the minimum (resp. maximum) value of density over the domain at the last iteration, and $\rho_{0}$ is the inflow density.

\begin{tabular}{c|c|c|c|c} 
name & $\begin{array}{c}\text { fluctuation } \\
\text { ceiling }\end{array}$ & $\begin{array}{c}\text { internal velocity } \\
\text { profile }\left(y^{+}<92\right)\end{array}$ & $\begin{array}{c}\text { recycling } \\
\text { distance }\end{array}$ & $\begin{array}{c}\text { density } \\
\text { lluctuations }\end{array}$ \\
\hline EIP ref. & yes & Van Driest & $2.2 \delta$ & $2.23 \%$ \\
EIP 10 & yes & Van Driest & $10 \delta$ & $5.15 \%$ \\
EIP no ceil. & no & Van Driest & $2.2 \delta$ & $11.38 \%$ \\
EIP raw & yes & linear & $2.2 \delta$ & $3.27 \%$
\end{tabular}

Table 1: EIP simulation parameters, and amplitude of density fluctuations.

The reference simulation is 'EIP ref.'. Comparing the simulations 'EIP $10 \delta$ ' and 'EIP 
ref.' shows a reduction of the recycling distance results in a reduction of the fluctuation intensity. This justifies the use of a recycling distance of only $2.2 \delta$ in the reference simulation. The effectiveness of the fluctuation ceiling at the inflow (cf. Eq.(1)) is also investigated: when it is switched off (simulation 'EIP no ceil.'), fluctuations are notably more intense than in 'EIP ref.'. Finally, the mean velocity profile at inflow has also an influence on the fluctuations: the simulation 'EIP raw', with the linear profile at $y^{+} \leq 92$, shows more intense fluctuations than 'EIP ref.', for which a more physical Van Driest profile is used.

Comparison of the simulations The friction coefficient of the different simulations is plotted in Fig.11. The reference simulation (EIP ref.) develops the expected decreasing tendancy the most rapidly, in less than $10 \delta\left(x_{1}\right)$. The use of a more crude mean velocity profile at inflow (EIP raw) delays the appearance of this behaviour, up to about $45 \delta\left(x_{1}\right)$. As observed previously, the longer recycling distance used in the simulation 'EIP $10 \delta$ ' results in more fluctuations, visible also on those space and time averaged results. Finally, particularly intense fluctuations are observed when the fluctuation ceiling is switched off in the recycling methodology (EIP no ceil.).

The mean and fluctuating velocity profiles at $x=x_{2}$ are shown in Fig.12. The four simulations yield a fairly good description of the mean velocity, with 'EIP raw' fitting best, surprisingly. Concerning the fluctuating velocity, the best results are achieved by the reference simulation. The other simulations overestimate $u^{\prime}$, in relation with the development of artificial periodic fluctuations, as previously evaluated in table 1 .

Reference results The mean-velocity profiles from the experiment and the reference simulation are compared in Fig.13, at the three axial positions. At $x_{1}$, in black, the simulated profile is constrained by the boundary condition and matches the corrected experimental profile. At $x_{2}$ and $x_{3}$, the velocity is slightly over-estimated in the logarithmic region. However, a good match is observed in the external region of the boundary layer, with a good prediction of the boundary layer thickening.

The fluctuating velocity in the axial direction, $u^{\prime}$, is also plotted in Fig.13. At $x_{1}$, the fluctuation profile is a result of the recycling method. It is slightly over-estimated with respect to the experimental profile. At $x_{2}$ and $x_{3}$, a peak develops around $y^{+}=20$. This is a classical feature of flat plate boundary layers, but the hot-wire measurements are 

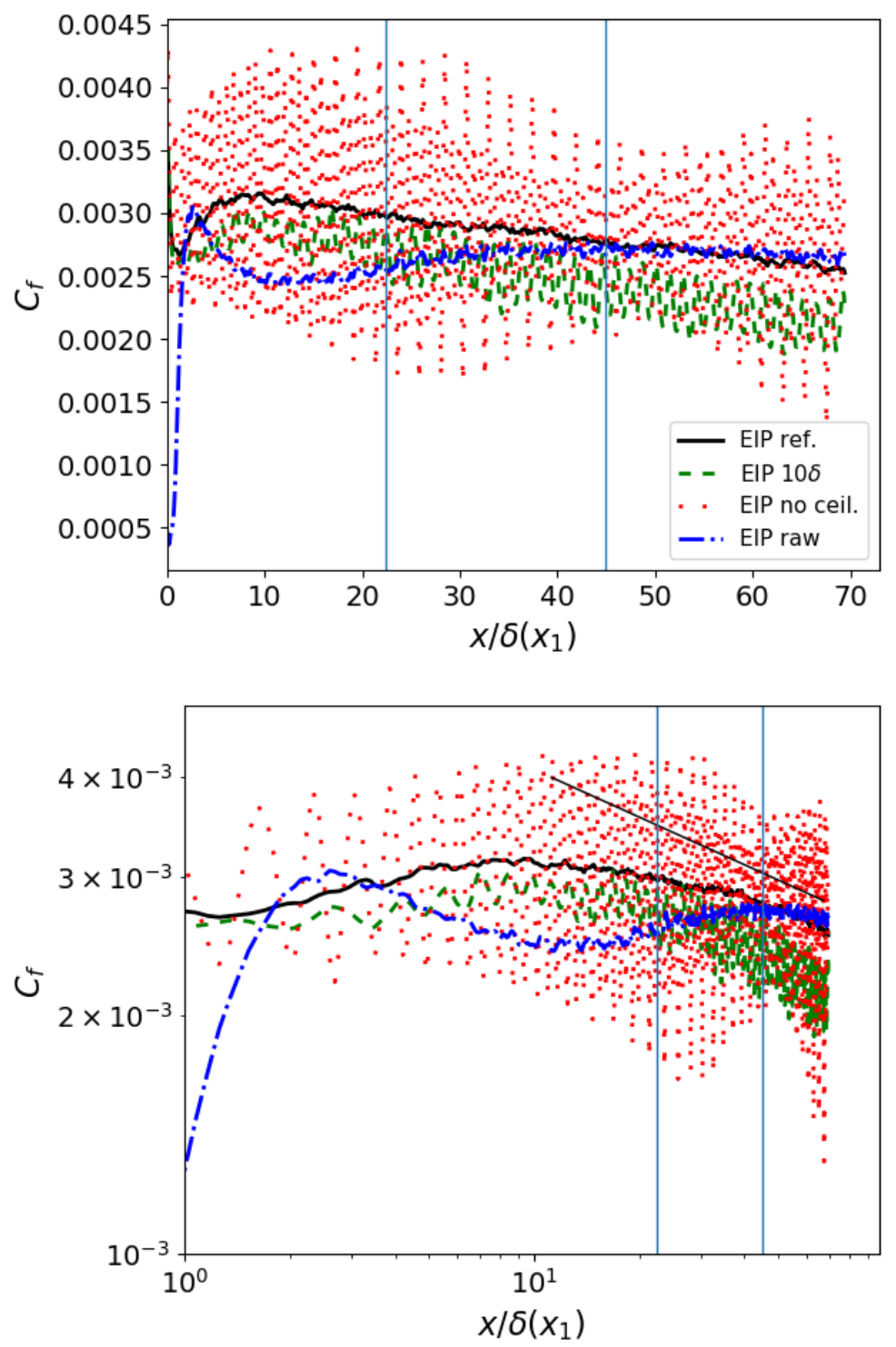

Figure 11: EIP case: streamwise evolution of the friction coefficient, for the different simulations, with linear and logarithmic scales. The thin vertical lines indicate the measurement sections at $x=x_{2}$ and $x=x_{3}$. The oblique thin line in the logarithmic plot indicates the $x^{-1 / 5}$ slope.
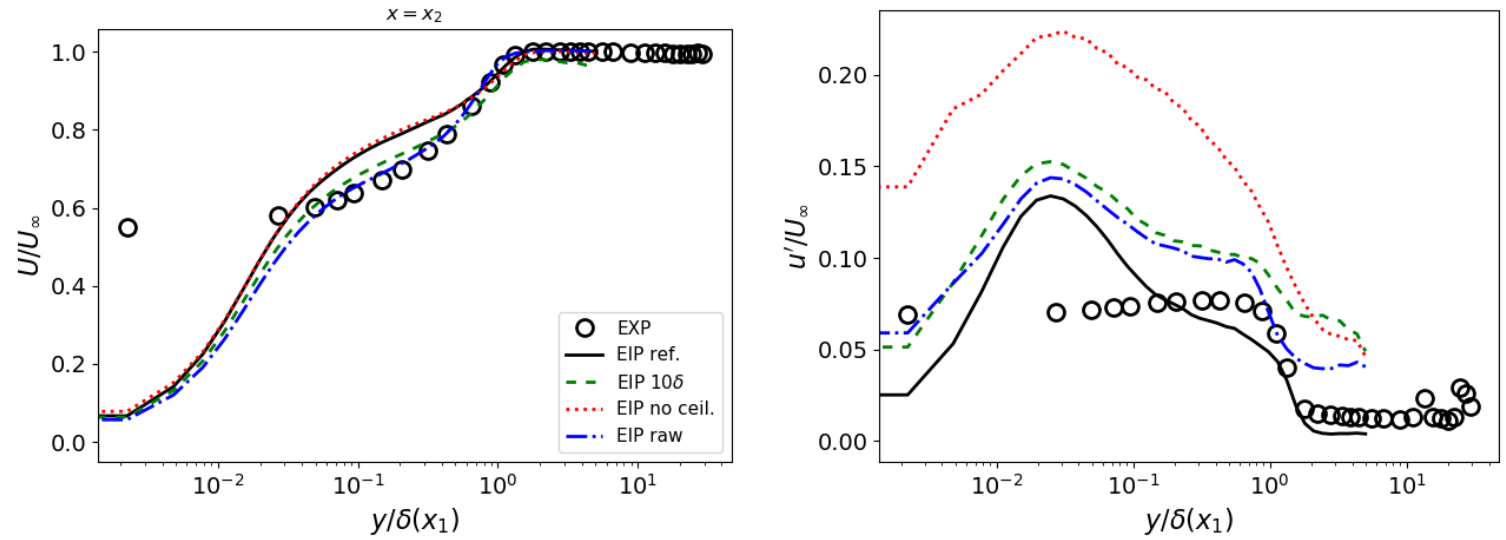

Figure 12: EIP case: mean velocity $(U)$ and fluctuating axial velocity $\left(u^{\prime}\right)$, for the different simulations, at $x=x_{2}$. Symbols: experiment, lines: simulations. 

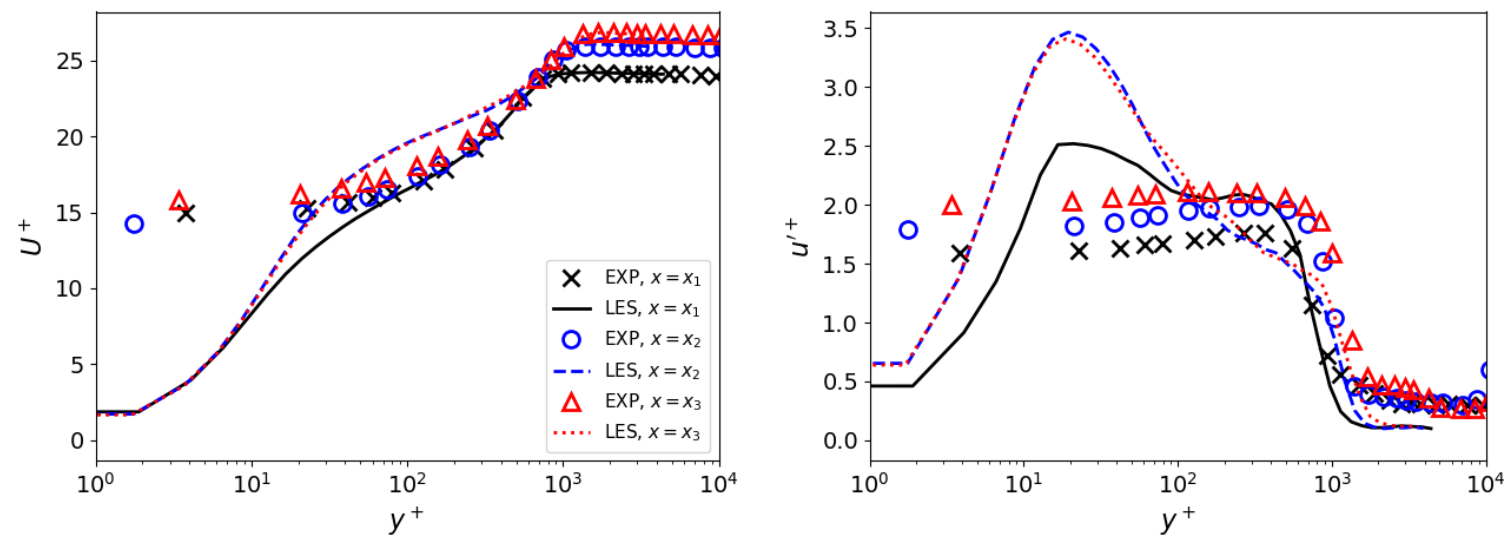

Figure 13: EIP case: mean velocity $(U)$ and fluctuating axial velocity $\left(u^{\prime}\right)$, at three axial positions. Symbols: experiment, lines: simulation (EIP ref.).

probably flawed in this region close to the wall. The fluctuations in the external region of the boundary layer, and the thickening of the boundary layer, are properly captured by the simulation.

\section{Conclusion}

A methodology to generate turbulent fluctuations in a boundary layer at the inflow of a large-eddy simulation has been investigated. It uses a recycling strategy: fluctuations extracted from a downstream source plane are rescaled and prescribed at the inflow, benefiting from the natural development of the turbulent eddies by the solver in the volume in-between. The present methodology is based on literature, but some features have been introduced, such as a running average of the mean profiles, or a ceiling of the amplitude of the velocity fluctuations. Moreover, the method has been evaluated in various conditions: incompressible or compressible flows, 3D boundary layer, different recycling distances or different levels of resolution of the prescribed mean velocity profile.

The different cases investigated show the friction coefficient can be recovered after a rather short distance $\left(<10 \delta\right.$, for $\left.\operatorname{Re}_{\theta}=2340\right)$. This indicates coherent turbulent eddies develop rapidly. Also, the mean and fluctuating velocity profiles are in good agreement with the reference profiles. Moreover, the methodology appears also effective in compressible conditions $(M=0.8)$ and with a 3D boundary layer. It shows some robustness regarding the resolution of the prescribed mean velocity profile. Concerning the recycling distance (i.e. distance between the target and source planes), values between $2 \delta$ and $10 \delta$ appear appropriate. However, values in the upper range seem to improve the development 
of the turbulent boundary layer in the compressible case investigated.

The main limitation of this recycling approach lies in the development of artificial fluctuations, whose main wavelength equals the recycling distance. In the present paper, a ceiling has been introduced on the amplitude of the inlet velocity fluctuations, which limits the amplitude of the fluctuations. However, depending on the case, those fluctuations can remain significant and could be problematic, for example in the context of acoustic studies. Future work should address this aspect.

\section{Nomenclature}

EIP simulations with an "experimental inflow profile"

$M \quad$ Mach number

NIP simulations with a "numerical inflow profile"

$R e_{x} \quad$ Reynolds number based on abscissa and external velocity

$u_{i} \quad$ instantaneous components of velocity $(i \in\{1,2,3\})$

$u_{i}^{\prime} \quad$ fluctuating components of velocity $(i \in\{1,2,3\})$

$u_{w} \quad$ wall friction velocity

W inner/outer boundary-layer regions weighting

$x_{1} \quad$ recycling inlet position

$\beta \quad$ rescaling factor

$\delta \quad$ boundary layer thickness

$\delta^{*} \quad$ displacement thickness

$\theta \quad$ momentum thickness

src [subscript] source section

tgt [subscript] target section (inflow)

$\ldots \quad$ mean

$<.>$ lateral spatial average

\section{Acknowledgments}

Computing resources have been provided by FLMSN-PMCS2I at Ecole Centrale de Lyon. 


\section{References}

[1] X. Wu, "Inflow turbulence generation methods," Annual Review of Fluid Mechanics, vol. 49, pp. 23-49, 2017.

[2] F. Gao, W. Ma, G. Zambonini, J. Boudet, X. Ottavy, L. Lu, and L. Shao, "Largeeddy simulation of 3-D corner separation in a linear compressor cascade," Physics of Fluids (1994-present), vol. 27, p. 085105, Aug. 2015.

[3] N. Jarrin, S. Benhamadouche, D. Laurence, and R. Prosser, "A synthetic-eddymethod for generating inflow conditions for large-eddy simulations," International Journal of Heat and Fluid Flow, vol. 27, no. 4, pp. 585-593, 2006.

[4] M. Pamiès, P.-É. Weiss, E. Garnier, S. Deck, and P. Sagaut, "Generation of synthetic turbulent inflow data for large eddy simulation of spatially evolving wall-bounded flows," Physics of Fluids, vol. 21, no. 4, p. 045103, 2009.

[5] A. Saghafian, L. Shunn, D. A. Philips, and F. Ham, "Large eddy simulations of the hifire scramjet using a compressible flamelet/progress variable approach," Proceedings of the Combustion Institute, vol. 35, no. 2, pp. 2163-2172, 2015.

[6] J. W. Kim and S. Haeri, "An advanced synthetic eddy method for the computation of aerofoil-turbulence interaction noise," Journal of Computational Physics, vol. 287, pp. 1-17, 2015.

[7] B. Xie, F. Gao, J. Boudet, L. Shao, and L. Lu, "Improved vortex method for largeeddy simulation inflow generation," Computers \& Fluids, vol. 168, pp. 87-100, May 2018.

[8] X. Wu, K. D. Squires, and T. S. Lund, "Large eddy simulation of a spatiallydeveloping boundary layer," in Proceedings of the 1995 ACM/IEEE conference on Supercomputing, p. 67, ACM, 1995.

[9] T. S. Lund, X. Wu, and K. D. Squires, "Generation of Turbulent Inflow Data for Spatially-Developing Boundary Layer Simulations," Journal of Computational Physics, vol. 140, pp. 233-258, Mar. 1998.

[10] S. Stolz and N. A. Adams, "Large-eddy simulation of high-Reynolds-number supersonic boundary layers using the approximate deconvolution model and a rescaling and recycling technique," Physics of Fluids (1994-present), vol. 15, pp. 2398-2412, Aug. 2003. 
[11] P. Sagaut, E. Garnier, E. Tromeur, L. Larcheveque, and E. Labourasse, "Turbulent Inflow Conditions for Large-Eddy Simulation of Compressible Wall-Bounded Flows," AIAA Journal, vol. 42, no. 3, pp. 469-477, 2004.

[12] A. Cahuzac, J. Boudet, P. Borgnat, and E. Lévêque, "Smoothing algorithms for mean-flow extraction in large-eddy simulation of complex turbulent flows," Physics of fluids, vol. 22, no. 12, 2010.

[13] J. Boudet, J.-F. Monier, and F. Gao, "Implementation of a roughness element to trip transition in large-eddy simulation," Journal of Thermal Science, vol. 24, Jan. 2015.

[14] H. L. Dryden, "Transition from laminar to turbulent flow," in Turbulent Flows and Heat Transfer, no. V in High Speed Aerodynamics and Jet Propulsion, Princeton University Press: C. C. Lin, 1959.

[15] J. Cousteix, Turbulence et couche limite. Cepadues editions, 1989.

[16] F. Nicoud and F. Ducros, "Subgrid-Scale Stress Modelling Based on the Square of the Velocity Gradient Tensor," Flow, Turbulence and Combustion, vol. 62, pp. 183-200, Sept. 1999.

[17] M. C. Jacob, E. Jondeau, and B. Li, "Time-resolved PIV measurements of a tip leakage flow," International Journal of Aeroacoustics, vol. 15, no. 6-7, pp. 662-685, 2016.

[18] J. Boudet, J. Caro, B. Li, E. Jondeau, and M. Jacob, "Zonal large-eddy simulation of a tip leakage flow," International Journal of Aeroacoustics, vol. 15, pp. 646-661, Oct. 2016.

[19] M. P. Simens, J. Jiménez, S. Hoyas, and Y. Mizuno, "A high-resolution code for turbulent boundary layers," Journal of Computational Physics, vol. 228, pp. 42184231, June 2009. 